\title{
Promotion of Family-Centered Birth With Gentle Cesarean Delivery
}

\author{
Susanna R. Magee, MD, MPH, Cynthia Battle, PhD, John Morton, MD, \\ and Melissa Nothnagle, MD, MSC
}

Purpose: In this commentary we describe our experience developing a "gentle cesarean" program at a community hospital housing a family medicine residency program. The gentle cesarean technique has been popularized in recent obstetrics literature as a viable option to enhance the experience and outcomes of women and families undergoing cesarean delivery.

Methods: Skin-to-skin placement of the infant in the operating room with no separation of mother and infant, reduction of extraneous noise, and initiation of breastfeeding in the operating room distinguish this technique from traditional cesarean delivery. Collaboration among family physicians, obstetricians, midwives, pediatricians, neonatologists, anesthesiologists, nurses, and operating room personnel facilitated the provision of gentle cesarean delivery to families requiring an operative birth.

Results: Among 144 gentle cesarean births performed from 2009 to 2012 , complication rates were similar to or lower than those for traditional cesarean births. Gentle cesarean delivery is now standard of care at our institution.

Conclusion: By sharing our experience, we hope to help other hospitals develop gentle cesarean programs. Family physicians should play an integral role in this process. ( $\mathrm{J}$ Am Board Fam Med 2014; 27:690-693.)

Keywords: Birth, Breast Feeding, Cesarean Section, Family, Mother-Infant Interaction

Despite research showing the importance of early mother-infant contact, cesarean delivery practices, including separation of the newborn from the mother, have not changed substantially over the past 30 years. A 1980 study of cesarean delivery comparing early mother-infant bonding with delayed contact showed improved self-assessment of mothering abilities and improved caretaking behavior (judged by blinded observers and sustained for several months) among dyads with early contact. ${ }^{1}$

This article was externally peer reviewed.

Submitted 14 January 2014; revised 23 May 2014; accepted 2 June 2014.

From the Department of Family Medicine, Memorial Hospital of Rhode Island, Providence (SRM, MN); Butler Hospital, Providence, RI (CB); Women \& Infants' Hospital of Rhode Island, Providence (CB); and Warren Alpert Medical School, Brown University, Providence, RI (SRM, CB, $\mathrm{JM}, \mathrm{MN})$.

Funding: none.

Conflict of interest: none declared.

Corresponding author: Susanna R. Magee, MD, MPH, Department of Family Medicine, Brown University, Memorial Hospital of Rhode Island, 111 Brewster St., Pawtucket, RI 02860 (E-mail: susannamagee@gmail.com).
Terms such as gentle cesarean or natural cesarean have been applied to a more patient- and family-centered approach to operative delivery. While gentle cesareans may comprise different techniques across institutions, a central feature is immediately placing the newborn skin-to-skin on the mother's chest and avoiding separation during or immediately after the cesarean. Early skin-to-skin contact has numerous documented benefits in the context of vaginal birth, including improved maternal perception of the birth experience ${ }^{2,3}$ and maternal-infant bonding, ${ }^{4}$ stability of the infant's temperature ${ }^{4-6}$, and initiation and duration of breastfeeding. ${ }^{7-9}$ With expertise in maternity and newborn care, surgical practices, and evidence-based medicine, family physicians are uniquely positioned to champion early motherinfant skin-to-skin contact, or gentle cesarean. In this commentary we describe our experiences over the past 5 years with developing and implementing a gentle cesarean program at a community hospital setting that houses a family medicine residency training program. Although these techniques have not yet been formally evaluated in the context of an 
empirical trial, our experiences to date suggest this approach may be promising and warrants further examination.

\section{Program Description}

To our knowledge, the gentle cesarean program founded at our hospital in 2009 is the first in the United States to implement this technique as the standard of care. Memorial Hospital of Rhode Island, a community hospital affiliated with Brown University, provides care to an urban underserved community and sponsors a family medicine residency. Approximately 500 births occur annually and are attended by family physicians, obstetricians, and midwives. Several family physicians have privileges for cesarean delivery. Patients experiencing preterm labor before 34 weeks' gestation are transferred to a local tertiary care center; however, transfer of other common obstetrical complications such as diabetes and hypertensive disorders is uncommon.

In 2009, a statewide advocacy group approached attending family physicians and obstetricians regarding strategies for improving family experiences of cesarean deliveries and promoting early skin-toskin contact. Because the available data suggested only benefits and no known adverse outcomes from immediate skin-to-skin contact, we decided to redesign our cesarean delivery practices. A team of providers developed a written protocol for what we refer to as "gentle cesarean," drawing on existing strategies for improving patient experiences of cesareans, ${ }^{3}$ as well as our team's expertise regarding strategies likely to have the most impact in our setting. Stakeholders, including representatives from family medicine, pediatrics, obstetrics, anesthesia, and nursing, then met to discuss practical elements of implementation. In the early stages we conducted gentle cesarean only for scheduled surgical cases and included the following elements: (1) minimal extraneous conversation among caregivers in the operating room; (2) music of the parents' choice playing, if desired; (3) delayed cord clamping for a minimum of 30 seconds after birth and stimulation of the infant (initial resuscitative measures) on the operating table before the cord is clamped and cut; (4) immediate placement of the infant skin-to-skin with the mother if both are clinically stable; (5) encouragement of early breastfeeding; and (6) avoidance of separation of mother and infant unless clinically indicated or desired by the mother.

Successful implementation of gentle cesarean required a systematic process of input and revision from relevant stakeholders. Initial concerns from the anesthesia department included positioning of the equipment, the support people (father, partner), and the infant to ensure appropriate access to continually and easily assess maternal stability and anesthesia needs. We were able to address these concerns through simulated delivery scenarios involving all team members.

Neonatal caregivers (nurses, family physicians, and pediatricians) were concerned about newborn hypothermia, given the typically cool ambient temperatures in the operating room for infection control. Although published literature allayed this concern, ${ }^{4-6}$ the team agreed to use frequent monitoring of maternal and infant temperature and preoperative and intraoperative maternal warming using equipment readily available in any operative suite (we use a "Baer hugger" warming device). In addition, representatives from all key departments agreed that the physician designated to receive the infant at the operating table would determine appropriateness for skin-to-skin placement based on his or her immediate clinical assessment of vigorousness. Vigorous infants are defined as those with a heart rate $>100 \mathrm{bpm}$, normal tone, and normal respirations and are qualified for immediate skin-to-skin contact. ${ }^{10}$

Because our model of gentle cesarean requires a dedicated neonatal provider to be present in the operating room at all times, nursing representatives pointed out the potential difficulty in obtaining needed supplies located outside the operative suite. We addressed this concern by creating a materials checklist for nurses so that the room would be properly equipped. In addition, we made it part of the gentle cesarean policy to have a resident physician and nurse in the operating room together for the remainder of the surgery after the birth. This ensured that the dyad would never be unattended if one staff member had to leave the operating suite for any reason.

After addressing stakeholder concerns, we held an orientation and training for all relevant members of the health care team; during this orientation, we described the rationale and potential benefits of gentle cesarean and addressed questions. Through an interactive demonstration, all team 
members learned about the new procedures and the proposed changes in the operating room environment for gentle cesarean.

Our first case, piloted on a scheduled repeat cesarean birth in 2009, resulted in a healthy and uncomplicated delivery, as well as a high level of patient satisfaction. However, this case also brought to light some additional minor logistical issues. This first case helped us determine that the anesthesia equipment needed to be moved slightly to accommodate the mother's support person(s) on her right side. This also allowed greater space for the physician's continued assessment of the infant on the mother's chest. This and other early cases also highlighted the importance of clinicians need to learn how to examine babies in a prone position. We also adjusted positions of intravenous poles and operating table armrests to make more physical space for staff and family members. To provide more space for the infant on the mother's chest, the anesthesia personnel now move the vertical drape separating them from the operating field from the maternal chest level toward the abdomen after the baby is born. Furthermore, nurses opted to change the placement of intravenous catheters from the antecubital fossa to a more convenient place on the nondominant arm so that it would not be obstructed when holding an infant.

\section{Assessment}

Between 2009 and 2012, we performed 144 gentle cesareans. The majority (68\%) of the cases were planned, and none were considered emergent. There were 18 cases of transient low infant temperature $\left(<97.4^{\circ}\right.$ axillary $)$ in the first hour of life; the majority occurred in the first 6 months of our program. In all cases, temperatures normalized with routine warming techniques for the infant, with no adverse consequences. After adding the perioperative warming blanket to maintain normal maternal temperature in the operating room before placing the infant skin to skin, no further instances occurred. In this case series we noted 11 cases of postoperative maternal fever, defined as a temperature $>100.4$. At $7.6 \%$, this rate is slightly lower than that of traditional cesarean delivery at our institution in the prior 3 years $(11.9 \%$, or 15 of 126).

Over time, our process of gentle cesarean has continued to evolve. Doulas (nonmedical labor support personnel) may enter the operating room along with the mother's birth partner. Although the first cases of gentle cesarean were for scheduled repeat cases, we implemented gentle cesarean in our nonscheduled or urgent cesareans starting in 2011, recognizing the potential for false-positive fetal monitoring and the probability of a healthy infant at birth, even in the case of a persistent category II fetal heart tracing. ${ }^{11,12}$ If newborns require suctioning for meconium at birth because they are not vigorous, we do this procedure at the warmer. If the infant becomes vigorous after suctioning and stimulation, we place then the infant skin-to-skin with the mother. The only scenario in which skin-to-skin is deemed impossible is the stat cesarean in which general anesthesia is required for the mother. This happens rarely, in $<1 \%$ of deliveries. Immediate skin-to-skin contact after cesarean has become the standard of care at our hospital.

As community awareness of our institution's approach to all cesarean deliveries has increased, patients who know during the prenatal period that they will require a cesarean delivery have transferred care to us to ensure the chance to have a gentle cesarean. In addition, women who have lower-risk pregnancies and are not expecting a cesarean delivery also are transferring care to us; they want to be sure that if a cesarean becomes necessary, their infant will get the chance to be with them immediately. Anecdotal feedback from patients has been very positive, reporting that, rather than experiencing an operation, patients and families were experiencing a birth.

It must be mentioned that a patient-centered approach to cesarean delivery should not be used to promote elective cesarean birth. We agree that vaginal birth is generally the safest mode of delivery, and each cesarean birth increases maternal risk during subsequent pregnancies. ${ }^{13}$ Yet when cesarean delivery is medically necessary, gentle cesarean may provide a positive birth experience for a family, rather than a surgery to be endured. Furthermore immediate skin-to-skin contact promotes early and successful breastfeeding, which may be adversely affected by cesarean birth compared with vaginal birth. ${ }^{14}$

\section{Conclusions}

Given recommendations for exclusive breastfeeding for 6 months and initiation of breastfeeding within 60 minutes of life, ${ }^{15-17}$ family physicians 
should consider advocating for changing the standard practice of separating infants from mothers in US cesarean deliveries. Based on our 5 years of practical experience, we believe gentle cesarean birth is a promising technique that may promote early mother-child bonding and successful breastfeeding, along with its associated improved health outcomes. Because this was not an empirical study, future research should evaluate the extent to which gentle cesarean programs supports these important family health outcomes. Given their expertise in birth and newborn care, family physicians are uniquely poised to promote gentle cesarean.

\section{References}

1. McClellan MS, Cabianca WA. Effects of early motherinfant contact following cesarean birth. Obstet Gynecol 1980;56:52-5.

2. DiMatteo MR, Morton SC, Lepper HS, et al. Cesarean childbirth and psychosocial outcomes: a metaanalysis. Health Psychol 1996;15:303-14.

3. Smith J, Plaat F, Fisk NM. The natural caesarean: a woman-centred technique. BJOG 2008;115:103742, discussion 1042.

4. Bystova K, Widström A, Matthiesen AS, et al. Skinto-skin contact may reduce negative consequences of "the stress of being born": a study on temperature in newborn infants, subjected to different ward routines in St. Petersburg. Acta Paediatr 2003;92:320-6.

5. Nolan A, Lawrence C. A pilot study of a nursing intervention protocol to minimize maternal-infant separation after cesarean birth. J Obstet Gynecol Neonatal Nurs 2009;38:430-42.

6. Gouchon S, Gregori D, Picotto A, Patrucco G, Nangeroni M, Di Giulio P. Skin-to-skin contact after cesarean delivery: an experimental study. Nurs Res 2010;59:78-84.

7. Moore ER, Anderson GC, Bergman N. Early skinto-skin contact for mothers and their healthy new- born infants (review). Cochrane Database Syst Rev 2007;18:CD003519.

8. Bramson L, Lee JW, Moore E, et al. Effect of early skin-to-skin mother-infant contact during the first 3 hours following birth on exclusive breastfeeding during the maternity hospital stay. J Hum Lact 2010;26: $130-7$.

9. Chalmers B, Kaczorowski J, Darling E, et al. Cesarean and vaginal birth in Canadian women: a comparison of experiences. Birth 2010;37:44-9.

10. American Academy of Pediatrics, American Heart Association. Neonatal resuscitation textbook, 6th ed. Elk Grove Village, IL: American Academy of Pediatrics; 2010.

11. Graham EM, Petersen SM, Christo DK, Fox HE. Intrapartum electronic fetal heart rate monitoring and the prevention of perinatal brain injury. Obstet Gynecol 2006;108(3 Pt 1):656-66.

12. Macones GA, Hankins GD, Spong CY, Hauth J, Moore T. The 2008 National Institute of Child Health and Human Development workshop report on electronic fetal monitoring: update on definitions, interpretation and research guidelines. Obstet Gynecol 2008;112:661-6.

13. Makoha FW, Felimban HM, Fathuddien MA, Roomi F, Ghabra T. Multiple cesarean section morbidity. Int J Gynaecol Obstet 2004;87:227-32.

14. Rowe-Murray HJ, Fisher JRW. Baby friendly hospital practices: cesarean section is a persistent barrier to early initiation of breast-feeding. Birth 2002;29: 124-31.

15. Section on Breastfeeding. Breastfeeding and the use of human milk. Pediatrics 2012;129:e827-841.

16. Protecting, promoting, and supporting breastfeeding: the special role of maternity services. A joint WHO/UNICEF statement. Int J Gynaecol Obstet 1990;31(Suppl 1):171-83.

17. Baby-Friendly USA. The guidelines \& evaluation criteria. Sandwich, MA: Baby-Friendly USA; 2010. Available from: http://www.babyfriendlyusa.org/getstarted/the-guidelines-evaluation-criteria. Accessed April 12, 2013. 